\title{
Resonant Oscillators with Carbon-Nanotube Torsion Springs
}

\author{
S. J. Papadakis, ${ }^{1 *}$ A. R. Hall, ${ }^{2}$ P. A. Williams, ${ }^{1}$ L. Vicci, ${ }^{3}$ M. R. Falvo, ${ }^{2}$ R. Superfine,${ }^{1,2,3}$ and S. Washburn ${ }^{1,2,3,4}$ \\ ${ }^{1}$ Department of Physics and Astronomy, University of North Carolina at Chapel Hill, Chapel Hill, North Carolina 27599, USA \\ ${ }^{2}$ Curriculum in Applied and Materials Science, University of North Carolina at Chapel Hill, \\ Chapel Hill, North Carolina 27599, USA \\ ${ }^{3}$ Department of Computer Science, University of North Carolina at Chapel Hill, Chapel Hill, North Carolina 27599, USA \\ ${ }^{4}$ Department of Biomedical Engineering, University of North Carolina at Chapel Hill, Chapel Hill, North Carolina 27599, USA
}

(Received 19 May 2004; published 27 September 2004)

\begin{abstract}
We report on the characterization of nanometer-scale resonators. Each device incorporates one multiwalled carbon nanotube (MWNT) as a torsional spring. The devices are actuated electrostatically, and their deflections, both low frequency and on resonance, are detected optically. These are some of the smallest electromechanical devices ever created and are a demonstration of practical integrated MWNT-based oscillators. The results also show surprising intershell mechanical coupling behavior in the MWNTs.
\end{abstract}

DOI: 10.1103/PhysRevLett.93.146101

PACS numbers: 68.65.-k, 81.07.-b

Paralleling the advances in electronic devices over the past few decades, recent fabrication advances have created ever smaller electromechanical devices and new applications for them. Nanometer-scale electromechanical devices [1-4] provide opportunities for high-density computational circuits and memories as well as high sensitivity sensors. One way to improve the performance of these devices is to reduce the oscillator effective mass while maintaining a high mechanical quality factor $(Q)$. We have fabricated nanometer-scale electromechanical oscillators each of which uses an individual multiwalled carbon nanotube (MWNT) as a torsional spring (Fig. 1). These devices are a demonstration of practical MWNTbased oscillators with possible applications as sensors, optical modulators, clocks for high-frequency electronics, etc.

Carbon nanotubes and other small objects like nanowires have been used in an electromechanical setting [511]. Here, we demonstrate self-contained, single-MWNT devices that do not require an electron microscope to detect the actuation. The devices are actuated electrostatically with small voltages, and their deflections are detected optically using interferometry, a method commonly used in current technologies. These are therefore a demonstration of practical MWNT-based devices.

MWNTs are an attractive candidate component for nanoelectromechanical systems (NEMS) due to their geometry, stiffness, and strength $[12,13]$. With diameters from a few to tens of nanometers, they allow the fabrication of devices with features smaller than the resolution limits of common lithographic techniques. It would be difficult lithographically to create uniform small highaspect-ratio suspended beams like the MWNT in Fig. 1. Furthermore, the current dominant material of microelectromechanical systems is silicon, the oxidation of which has been implied to limit devices' $Q$ [14]. MWNTs are chemically inert and do not suffer from the surface roughness and defects inherent in lithographically patterned NEMS. They therefore have the potential to have a high $Q[14,15]$. They also have large electrical and thermal conductivities. These features make them ideal candidates for use as springs in these devices.

Our device geometry is well suited for integration into electronic circuits. Unlike cantilever-type oscillators $[1,16,17]$, the paddle oscillator [18] allows current to be passed through the torsion spring to sense the deflection. Carbon nanotubes are promising in this regard, as their transport properties are likely to be affected more by strain than are those of a Si beam [19-21]. Thus, if these devices were to be used as sensors in which a stimulus causes a shift in the device resonance frequency $\left(F_{0}\right)$, they could be integrated into electronic circuits that monitor $F_{0}$ [1]. Another possible application is as clocks for highfrequency electronics. Currently, clocks use quartzcrystal resonators driven in an overtone mode, and use additional nonlinear circuits to generate harmonics at the desired frequency. With a nanometer-scale paddle oscillator, this could be done with a single-stage device. Our devices represent a significant step towards the realization of such integrated NEMS. The methods developed here could be applied to any rod-like objects such as nanowires, and are a step towards single-walled carbon nanotube-spring devices, which are likely to provide even better performance in the aforementioned applications.

The devices are fabricated as in Refs. [10,11]. Arc-grown [22] MWNTs are dispersed onto an oxidized Si wafer. Electron-beam lithography followed by deposition of $10 \mathrm{~nm}$ of $\mathrm{Cr}$ and $130 \mathrm{~nm}$ of $\mathrm{Au}$ is used to pattern large metal pads, the anchors, over the ends of each MWNT. A small metal paddle [18] with typical dimensions of about $600 \times 500 \mathrm{~nm}$, is patterned over the center of the MWNT. The paddle mass is typically somewhat less than $10^{-15} \mathrm{~kg}$ and the rotational moment of inertia is 


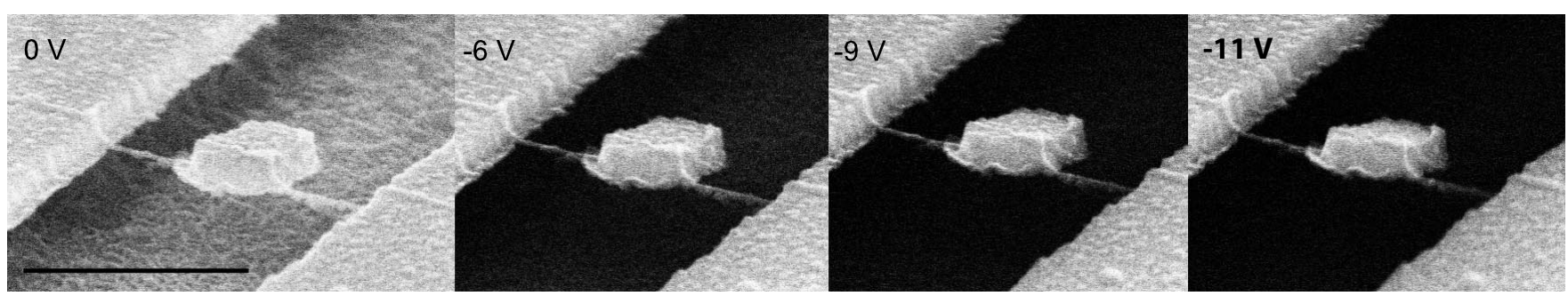

FIG. 1. Electrostatic deflection of device 9 in the SEM. The scale bar corresponds to $1 \mu \mathrm{m}$. The negative voltage is applied to the substrate, which causes the darkening of the substrate in the SEM images [24].

around $10^{-29} \mathrm{~kg} \mathrm{~m}^{2}$. Five hundred nanometers of the $\mathrm{SiO}_{2}$ is etched in hydroflouric acid to leave the paddle suspended, and supercritical $\mathrm{CO}_{2}$ is used to dry the sample.

Initial actuation tests are done in a scanning electron microscope (SEM) (Fig. 1). The paddle deflects and returns to its initial position after the voltage is returned to zero. Because of the asymmetry of the device (the paddle is not centered on the MWNT), the MWNT is strained primarily in torsion. When the paddle deflects the electrostatic force is concentrated at the end of the paddle farthest from the MWNT. In local-probe force measurements on similar devices, we found that vertical deflections of the MWNT were negligible compared to torsional deflections for a force applied away from the MWNT [10]. All of the devices we measure are significantly asymmetric, and except for device 10, the stretching or bending of the MWNT is negligible compared to the torsion.

Further measurements of both static [Fig. 2(b)] and oscillatory [Figs. 2(c) and 2(d)] deflections of the paddles are done interferometrically [Fig. 2(a)] [14,18,23]. Light scattered from the paddle interferes at the photodetector with light reflected from the fixed substrate and MWNT anchors. To measure the oscillatory deflections, a dc bias voltage plus a smaller ac drive voltage is applied to the substrate $[7,18,23]$. The ac component is generated by a spectrum analyzer, which is swept from lower to higher frequencies. The amplified photodiode signal is measured by the spectrum analyzer. Figures 2(c) and 2(d) show the resonance behaviors of two devices. The resonance frequencies $\left(F_{0}\right)$ of ten measured devices (Table I) cover a range from about 1 to $9 \mathrm{MHz}$ (our measurement apparatus rolls off above $10 \mathrm{MHz}$ ).

We calculate the torsional spring constant $K_{\theta}=$ $\left(2 \pi F_{0}\right)^{2} I$ of the MWNTs, estimating the rotational moment of inertia $I$ of each paddle from SEM images (Table I). Furthermore, for device 1, we also make a direct measurement of $K_{\theta}$ with an atomic force microscope (AFM) inside our SEM chamber [10]. We align the AFM tip over the end of the paddle and perform forcedistance measurements which yield the effective vertical spring constant of the point of contact of the AFM tip. From these measurements, the geometry of device 1, and the tip position, we calculate that $K_{\theta}=4.0 \pm 0.9 \times$ $10^{-15} \mathrm{Nm}$, consistent with the optical measurement [24].

Using $K_{\theta}$, the diameters, and the suspended lengths of the MWNTs, we also calculate their shear moduli. Since the details of the mechanical coupling between the shells of a particular MWNT are unknown, we calculate two shear moduli using models which represent the limits of what the intershell coupling might be [10]. The "solid rod model" assumes that the MWNT acts as a solid rod and yields a shear modulus $G_{e}$. The "outer shell model" assumes that there is no mechanical coupling between shells so only the outer shell of the MWNT is twisted when the paddle deflects and yields a shell shear modulus $G_{s}$. By comparing $G_{e}$ and $G_{s}$ to the expected shear modulus, $\sim 500 \mathrm{GPa}$, of carbon nanotubes [25], we can deduce how close to either limit a given MWNT is. We measure the MWNT diameter and the paddle $I$ to within about $20 \%$, which results in an estimated error of about a
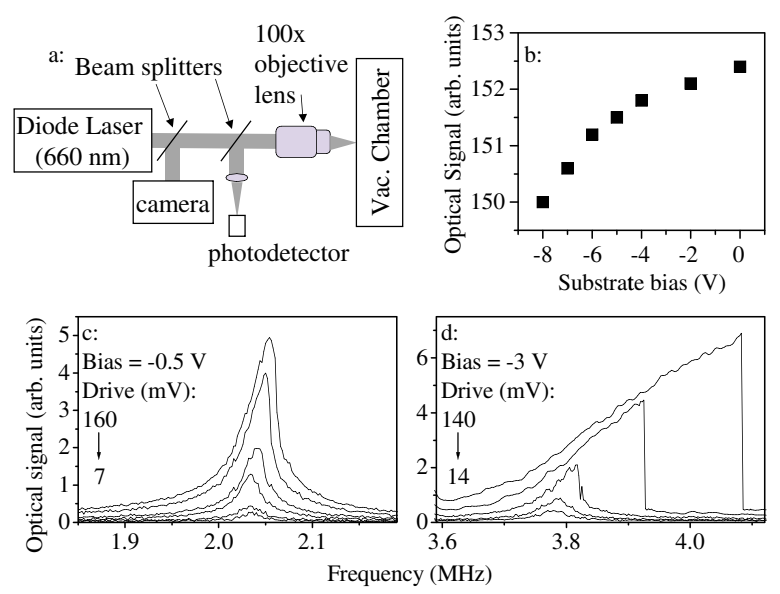

FIG. 2 (color online). (a) Schematic of the apparatus used to detect the paddle deflections. The photodetector signal is amplified by a transimpedance amplifier (gain $=20000$ ). The sample sits in a vacuum in the range of $10^{-5}$ Torr. The focused laser spot size is $\sim 0.7 \mu \mathrm{m}$. (b) Optically detected static deflection of device 9. Comparing this data to Fig. 1 demonstrates the sensitivity of the measurement technique. (c) and (d) Resonance behavior of devices 9 and 6, respectively. The bias is applied to the substrate. The drive voltage ranges listed are nominal output from a $50 \Omega$ source. 
factor of 2 for $G_{e}$ and $G_{s}$. For each device, comparing both $G_{e}$ and $G_{s}$ to the expected value of $\sim 500 \mathrm{GPa}$, we usually find that one of the values is unreasonably large or small. For devices 5-8 the solid rod model fits, but the outer shell model yields an impossibly large $G_{s}$. For devices 1-4 the outer shell model fits, but the solid rod model yields a very small $G_{e}$. Device 9 falls between the two limits. Surprisingly, most devices fall near one limit or the other, implying that, usually, a given MWNT has either negligible intershell mechanical coupling or strong enough coupling to prevent relative shell motion.

Previous investigators have observed that MWNT intershell coupling is typically initially weak $[10,26,27]$. In Ref. [10], similar devices stiffened due to an increase in intershell coupling caused by repeated deflections. For devices 5 and 9 we were able to drive the paddle to large enough amplitude that it tapped against the substrate, which was manifested as a clipping of the resonance peaks. This indicates a deflection of at least $45^{\circ}$. Since similar drive voltages were applied to the other devices and the geometries are similar, they likely were driven to comparable deflections, which correspond, depending on the device, to a shear strain of $\sim 2 \%$ to $5 \%$. This strain is larger than that which caused stiffening in Ref. [10]. Also, since they were driven on resonance, these devices have undergone many more deflections than those of Ref. [10]. One possible mechanism for increased intershell coupling is defects. It has been theoretically predicted that a few defects will increase the coupling by orders of magnitude [28]. Another is atomic registry between the shells, which may allow van der Waals forces to be strong enough to link them. Experiments show that a thin stripe of well registered atoms at the interface of a MWNT and a graphene sheet is sufficient to increase the force required for sliding by more than an order of magnitude [29]. The repeated straining of the MWNT might promote either of these mechanisms. However, whatever the mechanism, it is remarkable that it usually links all of the shells strongly or none at all. The results point out our lack of an understanding of the intershell mechanical coupling mechanisms.

All of the devices have resonance peak shapes that are qualitatively similar to those in Fig. 2, with peak asymmetries ranging from that in Fig. 2(c) to that in Fig. 2(d). Such peaks are typical of nonlinear oscillators $[14,18]$. There are multiple nonlinearities in this system. The shear strains of $\sim 2 \%$ to $5 \%$ are large enough that nonlinear behavior of the MWNT is expected [30]. The $\sim 45^{\circ}$ amplitude is large enough that the nonlinearity in the dependence of applied force on substrate voltage and paddle position are also significant. The optical intensity at the photodetector may also be a nonlinear function of the paddle deflection.

For half of the devices, the signal-to-noise ratio was large enough for us to observe symmetric peaks for small drive amplitudes, which became asymmetric at larger drive amplitudes. We concentrate now on the symmetric peaks, which indicate that the devices were operating in a linear regime. For those five devices we measure the mechanical quality factors $Q$ by fitting the solution of the driven weakly damped harmonic oscillator equation to the peaks (Table I).

One motivation for incorporating carbon nanotubes into electromechanical devices is the possibility, due to their surface perfection, of higher $Q$ than are achievable with $\mathrm{Si}$ devices of a similar size. As Si devices have shrunk, their $Q$ has also become smaller (see Figure 1 of Ref. [31]). While our devices are significantly smaller

TABLE I. Summary of MWNT torsional devices. $F_{0}$ is the resonance frequency, $K_{\theta}$ is the torsional spring constant of the MWNT, and $Q$ is the mechanical quality factor. $G_{s}$ and $G_{e}$ are shear moduli calculated using two models which represent the limits of what the MWNT mechanical properties might be. Devices 1-4 show weak intershell mechanical coupling while devices 5-8 display strong coupling (See text). The bold type indicates consistency with the expected shear modulus of nanotubes.

\begin{tabular}{|c|c|c|c|c|c|c|}
\hline Device & $\begin{array}{l}\text { Diameter } \\
(\mathrm{nm})\end{array}$ & $\begin{array}{c}F_{0} \\
(\mathrm{MHz})\end{array}$ & $\begin{array}{c}K_{\theta} \\
\left(10^{-14} \mathrm{Nm}\right)\end{array}$ & $\begin{array}{c}G_{s} \\
(\mathrm{GPa})\end{array}$ & $\begin{array}{c}G_{e} \\
(\mathrm{GPa})\end{array}$ & $Q$ \\
\hline 1 & 12 & 1.68 & $0.37(0.40)^{\mathrm{a}}$ & 570 & 90 & \\
\hline 2 & 27 & 2.37 & 1.6 & 460 & 35 & \\
\hline 3 & 28 & 2.50 & 1.9 & 740 & 54 & \\
\hline 4 & 35 & 3.27 & 3.6 & 560 & 33 & 78 \\
\hline 5 & 16 & 2.92 & 2.4 & 3420 & 435 & 81 \\
\hline 6 & 20 & 3.79 & 2.6 & 2880 & 290 & 140 \\
\hline 7 & 18 & 4.12 & 7.4 & 5000 & 565 & \\
\hline 8 & 22 & 2.98 & 3.9 & 2190 & 200 & \\
\hline 9 & 21 & 2.04 & 1.0 & 1200 & 120 & 93 \\
\hline $10^{\mathrm{b}}$ & 13 & 8.66 & & & & 103 \\
\hline
\end{tabular}

The $K_{\theta}$ in parentheses was measured directly using an atomic force microscope (See text).

${ }^{\mathrm{b}}$ This device displayed a vertical translational mode rather than a torsional mode. After processing, the paddle was perpendicular to the substrate so the torsional mode was not excited. The $F_{0}$ measured is consistent with what is predicted for the bending mode of the MWNT device using a Young's modulus of $\sim 1 \mathrm{TPa}$. 
than those plotted, their $Q$ of order 100 and flexing volumes of $\sim 10^{-13} \mathrm{~mm}^{3}$ fall in line with the trend. There are a few reasons our devices may not achieve the highest possible $Q$ for carbon-nanotube-based devices. First, we used arc-grown MWNTs in this work, which require post-growth purification with acids. This processing could introduce defects into the MWNTs. MWNTs grown by chemical vapor deposition directly onto the substrates would likely be less defective. Furthermore, we have not performed any cleaning steps after the etch. It is clear from our SEM images that in some cases there are impurities on the MWNTs (note the irregularities along the MWNT in Fig. 1) which would likely reduce $Q$. A vacuum system with a lower pressure might result in a higher measured $Q$. There are also fundamental factors that may limit $Q$. One is clamping losses where the MWNTs are attached to the anchors and the paddles. Different oscillator geometries might reduce these losses. Another is the intershell mechanical coupling. Our results show that the intershell coupling varies among the MWNTs, so it is likely that this coupling is due to some disordered form of bonding between the shells. If so, a reduction in $Q$ is expected. We are working towards single-walled carbon-nanotube-based devices that would not suffer from these losses.

Although higher- $Q$ devices should be achievable using carbon-nanotube springs, the current devices already show promise with their small size. For example, they approach the performance required to detect a single virus. If an adenovirus, with a mass of $3 \times 10^{-19} \mathrm{~kg}$, were to attach to the end of the paddle in device 6 , the frequency would shift by roughly $1 / 20$ th of the peak width. It is reasonable with the techniques we have used to fabricate paddles half the size of the ones we currently use, which would make them one eighth as massive and provide a significant increase in sensitivity.

We have demonstrated nanoelectromechanical torsional oscillators which use multiwalled carbon nanotubes as torsion springs. These are demonstrations of practical devices, as they are actuated with small voltages and detected optically, and are a crucial step in the development of self-contained nanometer-scale devices. The results also point to some of the challenges involved in making manufacturable devices with multiwalled nanotubes. The anticipated high $Q$ was not observed. It is likely that defects in the nanotubes and the imperfect cleanliness of the nanotube surfaces play a significant role in the dissipation, so better nanotube purification and sample fabrication techniques will be required. Furthermore, the intershell mechanical coupling varies significantly from one nanotube to the next, and is not controlled or well understood. These factors lead to difficulties in the design of a device with a desired resonance frequency. This is a parameter that must be controlled for the realization of manufacturable devices.
We thank Stephane Evoy for helpful advice and the ONR and NSF for funding for this project.

*Current address: Johns Hopkins University Applied Physics Laboratory, Laurel, Maryland 20723, USA

[1] H. G. Craighead, Science 290, 1532 (2000).

[2] A. Erbe, C. Weiss, W. Zwerger, and R. H. Blick, Phys. Rev. Lett. 87, 096106 (2001).

[3] A. N. Cleland and M. L. Roukes, Nature (London) 392, 160 (1998).

[4] Y.T. Yang et al., Appl. Phys. Lett. 78, 162 (2001).

[5] P. Poncharal, Z. L. Wang, D. Ugarte, and W. A. de Heer, Science 283, 1513 (1999).

[6] P. Kim and C. M. Lieber, Science 286, 2148 (1999).

[7] M.-F. Yu, G. J. Wagner, R. S. Ruoff, and M. J. Dyer, Phys. Rev. B 66, 073406 (2002).

[8] A. M. Fennimore et al., Nature (London) 424, 408 (2003).

[9] P. A. Williams et al., Appl. Phys. Lett. 80, 2574 (2002).

[10] P. A. Williams et al., Phys. Rev. Lett. 89, 255502 (2002).

[11] P. A. Williams et al., Appl. Phys. Lett. 82, 805 (2003).

[12] M. R. Falvo et al., Nature (London) 389, 582 (1997).

[13] E.W. Wong, P. E. Sheehan, and C. M. Lieber, Science 277, 1971 (1997).

[14] D. W. Carr et al., Appl. Phys. Lett. 75, 920 (1999).

[15] R. Lifshitz and M. L. Roukes, Phys. Rev. B 61, 5600 (2000).

[16] J. Fritz et al.,Science 288, 316 (2000).

[17] B. Ilic et al., Appl. Phys. Lett. 77, 450 (2000).

[18] S. Evoy et al.,J. Appl. Phys. 86, 6072 (1999).

[19] L. Yang, M. P. Anantram, J. Han, and J. P. Lu, Phys. Rev. B 60, 13874 (1999).

[20] J.W. Ding et al., J. Phys. Condens. Matter 15, L439 (2003).

[21] E. D. Minot, Phys. Rev. Lett. et al., 90, 156401 (2003).

[22] T.W. Ebbesen and P. M. Ajayan, Nature (London) 358, 16 (1992).

[23] D.W. Carr, L. Sekaric, and H. G. Craighead, J. Vac. Sci. Technol. B 16, 3821 (1998).

[24] See EPAPS Document No. E-PRLTAO-93-040438 for videos of the actuation depicted in Fig. 1 and of the AFM force-distance measurements of the torsional spring constant of device 1 . A direct link to this document may be found in the online article's HTML reference section. The document may also be reached via the EPAPS homepage (http:/www.aip.org/pubservs/epaps. html) or from ftp.aip.org in the directory /epaps/. See the EPAPS homeage for more information.

[25] J. P. Lu, Phys. Rev. Lett. 79, 1297 (1997).

[26] J. Cumings and A. Zettl, Science 289, 602 (2000).

[27] M.-F. Yu et al., Science 287, 637 (2000).

[28] M. Huhtala et al., Phys. Rev. B 70, 045404 (2004).

[29] M. R. Falvo et al., Nature (London) 397, 236 (1999); M. R. Falvo, J. Steele, R. M. Taylor II, and R. Superfine, Phys. Rev. B 62, R10665 (2000).

[30] M. Buongiorno Nardelli, B. I. Yakobson, and J. Bernholc, Phys. Rev. Lett. 81, 4656 (1998).

[31] P. Mohanty et al., Phys. Rev. B 66, 085416 (2002). 